\title{
Effect of the electron density stratification on the off-limb O VI line profiles
}

\author{
N.-E. Raouafi and S. K. Solanki \\ Max Planck Institut für Sonnensystemforschung, MPS, Katlenburg-Lindau, Germany \\ email: Raouafi@mps.mpg.de ; Solanki@mps.mpg.de
}

\begin{abstract}
Coronagraphic spectral observations carried out with UVCS on SoHO above polar coronal holes have shown that the velocity distribution is highly anisotropic. Here we examine the influence of the density stratification on the interpretation of such observations. In particular, we investigate the profiles of $\mathrm{O}$ vI spectral lines emitted in polar coronal holes. We find that at distances greater than $1 R_{\odot}$ from the solar surface the widths of the emitted lines are significantly affected by the details of the adopted electron density profiles. In particular, the densities deduced by Doyle et al. (1999) from SoHO data result in O vi profiles whose widths and intensity ratio are relatively close to the values observed by UVCS although only isotropic velocity distributions are employed. Hence we expect that the magnitude of the anisotropy of the velocity distribution deduced from UVCS data depends strongly on the adopted density profile.
\end{abstract}

\section{Introduction}

One of the most exciting results obtained from data recorded by UVCS is the large width of spectral lines emitted by heavy ions (namely $\mathrm{O}$ VI and $\mathrm{Mg} \mathrm{x}$ ) in polar coronal holes during solar activity minimum. In order to reproduce simultaneously the observed line widths and values of the intensity ratio between the $\mathrm{O}$ vi lines, a significant anisotropy in the velocity distributions of the heavy ions was invoked $\left(T_{\perp} / T_{\|}>10\right.$; Kohl et al. 1997; etc). This result has been interpreted in terms of ion-cyclotron waves propagating through the corona (Cranmer et al. 1999). In the present paper we consider the influence of the exact choice of the adopted density stratification on the $\mathrm{O}$ vi doublet in a polar coronal hole.

\section{Computed spectral lines and Atmospheric parameters}

We compute the intensity profiles of the O vi doublet ( $\lambda 1032$ and $\lambda 1037.6)$. We take into account the effect of the solar wind (Doppler dimming and optical pumping of $\mathrm{O}$ VI $\lambda 1037.6$ line by the $\mathrm{C}$ II doublet $\lambda 1036.3 \& \lambda 1037)$. The line profile is integrated along the line of sight and only an isotropic velocity distribution is considered. Two density profiles are considered (see Fig. 1). The importance of the density profile resides therein that it influences the line profile integrated along the LOS directly, but in addition also indirectly through the solar wind speed. The absolute outflow speed of the solar wind is prescribed at the base of the corona and is obtained at all locations of open flux from the mass and magnetic flux conservation equations (assuming that the flow follows the field lines).

\section{Results}

Fig. 2a shows that whereas line profiles calculated using the Guhathakurta \& Holzer (1994) density profile (plusses) are too narrow at large distances compared to UVCS 

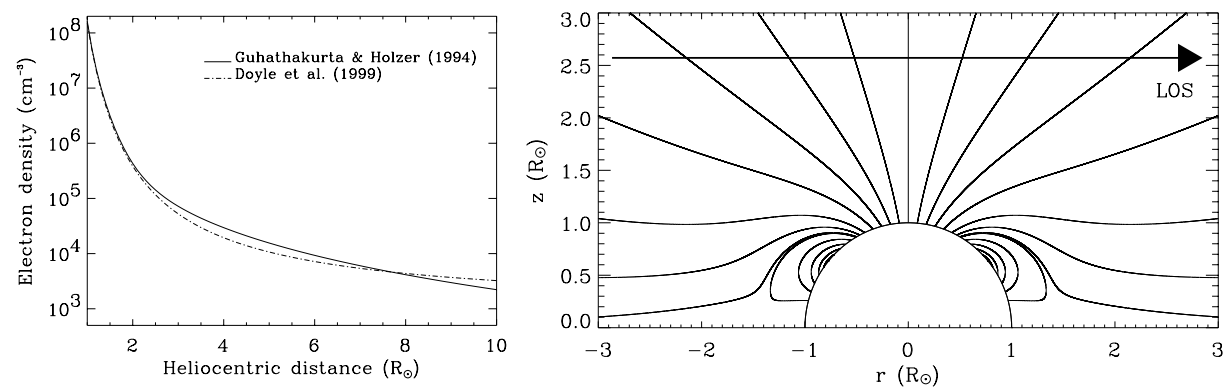

Figure 1. Left panel: Electron density given as a function of distance to the center of the Sun. Solid line: Guhathakurta \& Holzer (1994; polynomial fit); dot-dashed line: Doyle et al. (1999; exponential fit). Right panel: Adopted magnetic configuration (Banaszkiewicz et al. 1998).
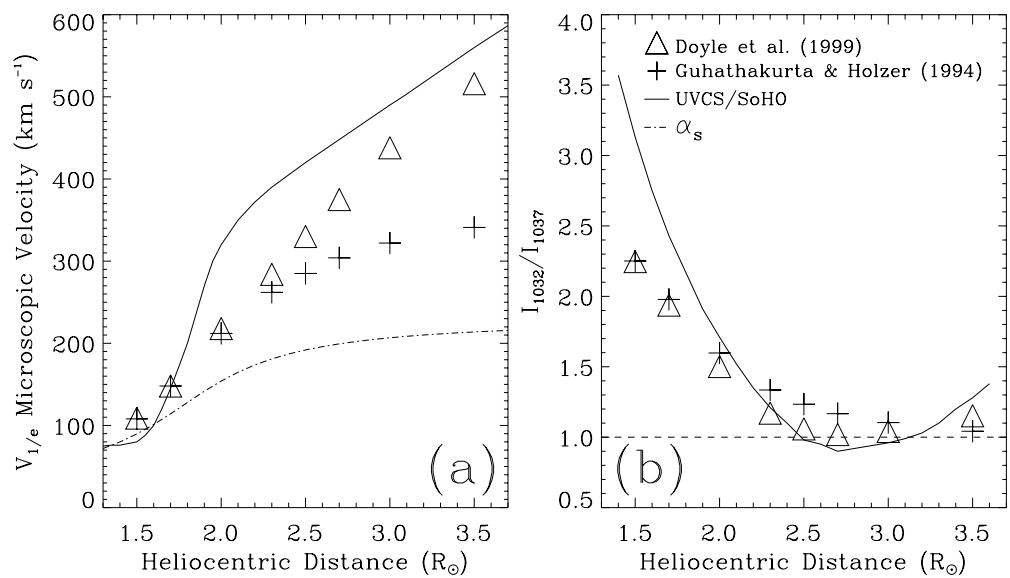

Figure 2. (a) The e-folding widths $\left(V_{1 / e}\right)$ and (b) total intensity ratios of the line-of-sight integrated profiles of the $\mathrm{O}$ vi doublet vs projected heliocentric distance.

observations (solid line), profiles based on the Doyle et al. (1999) density profile (triangles) have similar widths. Fig. $2 \mathrm{~b}$ shows that at heliocentric distances of 2.5 and $2.7 R_{\odot}$ the computed intensity ratio drops to very nearly unity, in general agreement with the UVCS observations.

\section{Discussion}

Our results demonstrate a clear need for a reappraisal of the importance of the anisotropy of the kinetic temperature of the heavy ions in the solar corona. This in turn has implications for the mechanisms of heating and acceleration of different species in the polar coronal holes. We are currently testing if the observed widths of the H I Ly- $\alpha$ and $\mathrm{Mg}$ x doublet lines are also roughly reproduced by the Doyle et al. (1999) density for an isotropic velocity distribution.

\section{References}

Banaszkiewicz M., Axford W. I., \& McKenzie J. F. 1998, A\&A, 337, 940

Cranmer, S. R., Kohl, J. L., Noci, G., et al. 1999, ApJ, 511, 481

Doyle, J. G., Teriaca, L., \& Banerjee, D. 1999, A\&A, 349, 956

Guhathakurta, M., \& Holzer, T. E. 1994, ApJ, 426, 782

Kohl, J. L., Noci, G., Antonucci, E., et al. 1997, Sol. Phys., 175, 613 